\title{
Fatores agravantes da Síndrome de Burnout nos profissionais de Enfermagem
}

\author{
Aggeavating factors of Burnout Syndrome in Nursing professionals \\ Factores agravantes del Síndrome de Burnout en profesionales de Enfermería
}

Maria Eduarda Wanderley de Barros Silva

ORCID: https://orcid.org/0000-0002-4642-3282

Universidade Federal de Campina Grande, Brasil

E-mail: eduarda.wanderley@outlook.com

Vitoria Vilas Boas da Silva Bomfim

ORCID: https://orcid.org/0000-0003-4897-0279

Centro Universitário Jorge Amado, Brasil E-mail: pesquisaclinica9@gmail.com

Eduarda Heloísa de Freitas Silva

ORCID: https://orcid.org/0000-0003-4898-5358

Universidade Federal de Pernambuco, Brasil E-mail: eduarda.hfsilva@ufpe.br

Mirely Marluce Soares da Silva

ORCID: https://orcid.org/0000-0003-3231-1033

Universidade Federal de Pernambuco, Brasil E-mail: mirely.marluce@ufpe.br

Luana Cristina da Silva

ORCID: https://orcid.org/0000-0001-6269-0068

Universidade Federal de Pernambuco, Brasil

E-mail: luana.cristina@ufpe.br

Ana Carolina do Nascimento

ORCID: https://orcid.org/0000-0002-8130-0819

Universidade Federal de Pernambuco, Brasil

E-mail: carolina.nascimento2@ufpe.br

Matheus da Silva Sales

ORCID: https://orcid.org/0000-0002-8724-1100

Universidade Federal de Pernambuco, Brasil

E-mail: tetheusales@gmail.com

Matheus Winicius Claudino Coutinho

ORCID: https://orcid.org/0000-0001-5410-011X Universidade Maurício de Nassau, Brasil

E-mail: mwccoutinho@gmail.com

Jean Scheievany da Silva Alves

ORCID: https://orcid.org/0000-0002-3693-3676

Universidade Federal de Pernambuco, Brasil E-mail: jean.alves@ufpe.br

Samara Dantas de Medeiros Diniz

ORCID: https://orcid.org/0000-0001-9418-0185

Faculdade Estácio do Rio Grande do Norte, Brasil

E-mail: samaradantas1998@hotmail.com

\section{Resumo}

A Síndrome do Esgotamento Profissional ou Síndrome de Burnout (SB), por sua vez, está relacionada ao estresse no trabalho em níveis que ultrapassam a possibilidade de adaptação. Dessa forma, o estresse ocupacional é um problema de saúde pública caracterizado por lesão de causa multifatorial, decorrente de relação entre trabalhador e seu ambiente. Trata-se de uma revisão integrativa da literatura. Sendo seguida as seguintes etapas identificação do tema e seleção da pesquisa, elaboração dos critérios de inclusão e exclusão, seleção dos estudos, categorização dos estudos selecionados, análise e interpretação dos resultados. Após a aplicação dos critérios de inclusão e exclusão, o corpus de análise foi composto por 14 artigos. Foi encontrado que a organização no trabalho exercia uma forte influência sobre o aparo psíquico dos profissionais de enfermagem podendo ocorrer após experiências negativas o desgaste gradual do sujeito resultando em um quadro sintomatológicos como a presença de ansiedade, depressão, cefaleia, agitação e irritabilidade. Assim é válido destacar que os sintomas aparecem de forma distinta em cada trabalhador. O estado emocional dos profissionais que trabalham em setores fechados altera-se no decorrer do plantão visto que pode estar associado ao desgaste e estresse da atividade de fornecer ao paciente assistência e cuidado, principalmente devido a existência de altos níveis de habilidade e conhecimento que exige resposta imediata. Os achados deste artigo mostram 
a necessidade de acompanhamento das condições de trabalho e saúde destes profissionais, além disso é preciso que se tenha uma implementação de estratégias que possam minimizar os danos à saúde dos mesmos.

Palavras-chave: Esgotamento profissional; Profissionais de enfermagem; Condições de trabalho.

\begin{abstract}
The Professional Burnout Syndrome or Burnout Syndrome (BS), in turn, is related to stress at work at levels that exceed the possibility of adaptation. Thus, occupational stress is a public health problem characterized by a multifactorial cause of injury, arising from the relationship between workers and their environment. This is an integrative literature review. The following steps are followed: identification of the topic and selection of the research, elaboration of inclusion and exclusion criteria, selection of studies, categorization of selected studies, analysis and interpretation of results. After applying the inclusion and exclusion criteria, the corpus of analysis consisted of 14 articles. It was found that the organization of work exerted a strong influence on the psychological impairment of nursing professionals, which may occur after negative experiences, the gradual wear of the subject resulting in a symptomatological picture such as the presence of anxiety, depression, headache, agitation and irritability. Thus, it is worth noting that the symptoms appear differently in each worker. The emotional state of professionals working in closed sectors changes during the shift as it may be associated with the wear and stress of the activity of providing the patient with assistance and care, mainly due to the existence of high levels of skill and knowledge that require a response immediate. The findings of this article show the need to monitor the working conditions and health of these professionals, furthermore, it is necessary to implement strategies that can minimize damage to their health.
\end{abstract}

Keywords: Professional burnout; Nursing professionals; Working conditions.

\title{
Resumen
}

El Síndrome de Burnout Profesional o Síndrome de Burnout (SB), a su vez, se relaciona con el estrés en el trabajo en niveles que superan la posibilidad de adaptación. Así, el estrés laboral es un problema de salud pública caracterizado por una causa multifactorial de lesión, que surge de la relación entre los trabajadores y su entorno. Ésta es una revisión integradora de la literatura. Se siguen los siguientes pasos: identificación del tema y selección de la investigación, elaboración de criterios de inclusión y exclusión, selección de estudios, categorización de estudios seleccionados, análisis e interpretación de resultados. Luego de aplicar los criterios de inclusión y exclusión, el corpus de análisis estuvo conformado por 14 artículos. Se encontró que la organización del trabajo ejerció una fuerte influencia en el deterioro psicológico de los profesionales de enfermería, que puede ocurrir luego de experiencias negativas, el desgaste gradual del sujeto resulta en un cuadro sintomatológico como la presencia de ansiedad, depresión, dolor de cabeza, agitación. e irritabilidad. Por tanto, cabe señalar que los síntomas aparecen de forma diferente en cada trabajador. El estado emocional de los profesionales que trabajan en sectores cerrados cambia durante el turno ya que puede estar asociado al desgaste y estrés de la actividad de brindar asistencia y cuidado al paciente, principalmente debido a la existencia de altos niveles de habilidad y conocimiento que requieren un respuesta inmediata. Los hallazgos de este artículo muestran la necesidad de monitorear las condiciones laborales y de salud de estos profesionales, además, es necesario implementar estrategias que puedan minimizar el daño a su salud.

Palabras clave: Burnout profesional; Profesionales de enfermería; Condiciones laborales.

\section{Introdução}

O termo Burnout, do inglês burn = queimar e out $=$ fora, possui como descrição o limite de suas forças, colapso da capacidade adaptativa do indivíduo e exaustão da emoção. A SB identificada na década de 1970, caracteriza-se por ser uma tríade de dimensões sendo exaustão emocional, despersonalização e redução da realização pessoal (Perniciotti et al., 2020).

A exaustão emocional (EE) é caracterizada por fadiga intensa e falta de forças para enfrentar o dia de trabalho e sensação de estar sendo exigido além de seus limites emocionais. A despersonalização (DE) possui como característica o distanciamento emocional e indiferença em relação ao trabalho. A redução da realização pessoal (RP) se expressa como falta de perspectivas para o futuro, frustração e sentimentos de incompetência e fracasso (Perniciotti et al., 2020).

O estresse é definido como uma resposta do indivíduo ao agente agressor pois aciona a reação de luta e fuga em uma tentativa de retornar ao estado de equilíbrio, sendo um conjunto de respostas fisiológicas específicas que podem gerar manifestações de ordem mental ou física. Assim, os transtornos mentais são caracterizados por sintomas de fadiga, irritabilidade, insônia, ansiedade e queixas somáticas (Sousa et al., 2015).

A Síndrome do Esgotamento Profissional ou Síndrome de Burnout (SB), por sua vez, está relacionada ao estresse no trabalho em níveis que ultrapassam a possibilidade de adaptação. Dessa forma, o estresse ocupacional é um problema de saúde 
pública caracterizado por lesão de causa multifatorial, decorrente de relação entre trabalhador e seu ambiente (Vieira \& Jane, 2021).

Estudos apontam que o Burnout entre os profissionais de enfermagem é mais elevado do que em outros profissionais de saúde. Pois as exposições contínuas a eventos ocupacionais que causam a SB apresentam uma série de sintomas psicossociais e físicos sendo cansaço, mau humor, irritabilidade e baixa produtividade. Nesse sentido o processo de trabalho dos profissionais de enfermagem é constituído por condições potencialmente estressantes, que podem afetar diretamente a sua saúde, bem como a qualidade da assistência prestada aos usuários (Vieira \& Jane, 2021).

Observa-se que essa categoria enfrenta maiores vulnerabilidade aos agentes causais de adoecimento no trabalho em virtude do envolvimento que possui com o paciente internado, da deficiência de recursos, jornadas longas e duplas de trabalho, pouco tempo de descanso, conflitos interpessoais, baixa remuneração, precária valorização profissional resultando em baixa autoestima e baixo desempenho profissional (Silva, 2006).

Ao considerar os impactos pessoais e ocupacionais da SB nos profissionais de enfermagem, torna-se relevante aprimorar as investigações acerca dessa temática. Desta forma, a presente revisão integrativa da literatura tem como objetivo identificar estudos que abordem os fatores agravantes da síndrome de Burnout nos profissionais de enfermagem.

\section{Metodologia}

Trata-se de uma revisão integrativa da literatura de abordagem qualitativa (Ludke \& Andre, 2013). Sendo seguida as seguintes etapas identificação do tema e seleção da pesquisa, elaboração dos critérios de inclusão e exclusão, seleção dos estudos, categorização dos estudos selecionados, análise e interpretação dos resultados (Souza et al., 2010).

Para a estratégia de busca foi utilizado os descritores pré-estabelecidos pelo Descritores em Ciências da Saúde (DECS) sendo "Esgotamento Profissional" e "Profissionais de Enfermagem". Tendo como pergunta definida e tema de interesse: "Quais os fatores agravantes da Síndrome de Burnout nos profissionais de enfermagem?".

Foram utilizadas as bases de dados PubMed, Literatura Latino-Americana e do Caribe em Ciências da Saúde (LILACS) e Scientific Eletronic Library Online (SciELO). A consulta ocorreu durante os meses de maio e junho de 2021.

As versões em português dos descritores compuseram as estratégias de busca a partir do cruzamento: I) Profissionais de Enfermagem AND Esgotamento Profissional. Ressalta-se que a estratégia foi aplicada conforme as características de cada base de dados.

A declaração PRISMA foi utilizada para impor avaliação crítica ao corpus de análise da pesquisa (Zoltowski et al., 2014). Os critérios de inclusão adotados foram: I) estudos clínicos e randomizados que respondessem à questão norteadora a partir da leitura do título e resumo; II) período de publicação entre os anos de 2015 a 2021; e III) estar nos idiomas português ou inglês. Os critérios de exclusão envolveram estudos duplicados e que correspondessem a revisão integrativa, livros, cartas ao editor e artigos nota prévia. Não foram delimitados idiomas.

\section{Resultados}

A estratégia de busca resultou em um total de 308 artigos. O Quadro 1 apresenta o quantitativo de resultados e a relação do cruzamento utilizado conforme a base de dados utilizada. 
Quadro 1: Quantitativo de resultados por base de dados e cruzamento. Brasil, 2021.

\begin{tabular}{|c|c|}
\hline Base de dados & $\begin{array}{c}\text { Profissionais de Enfermagem AND Esgotamento } \\
\text { Profissional }\end{array}$ \\
\hline LILACS & 256 \\
\hline PUBMED & 1 \\
\hline SCIELO & 51 \\
\hline Total & 308 \\
\hline
\end{tabular}

Fonte: Autores (2021).

A Figura 1 apresenta o fluxograma de seleção dos resultados encontrados. Para que se tenha a facilidade de compreensão de cada critério utilizado.

Figura 1: Fluxograma de seleção dos resultados da pesquisa segundo as recomendações PRISMA. Brasil, 2021.

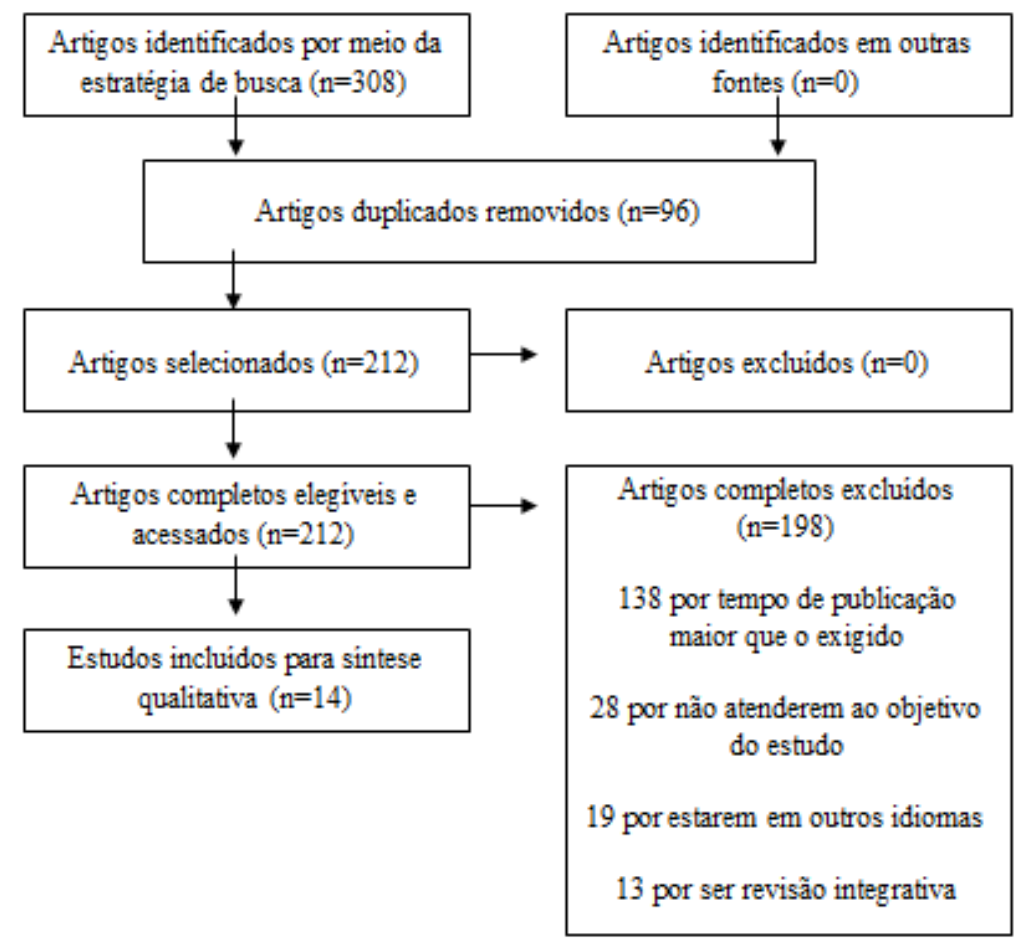

Fonte: Autores (2021).

Após a aplicação dos critérios de inclusão e exclusão, o corpus de análise foi composto por 14 artigos, dos quais 8 estavam indexados à SciELO e 6 à LILACS.

A não seleção de 291 artigos ocorreu por 138 possuírem tempo de publicação maior que o exigido (47\%), 96 por estarem duplicados (32,9\%), 28 por não atenderem ao objetivo do estudo (9,6\%), 19 por não estar nos idiomas selecionados $(6,5 \%)$ e 13 por ser revisão integrativa $(4 \%)$.

A aplicação da técnica de análise dos artigos foi realizada durante os meses de maio e junho de 2021. O Quadro 2 demonstra a caracterização do corpus conforme informações de autoria, ano de publicação, local do estudo, título do artigo, objetivo do estudo, delineamento metodológico e desfecho. 
Quadro 2: Caracterização do corpus de análise de pesquisa. Brasil, 2021.

\begin{tabular}{|c|c|c|c|c|}
\hline $\begin{array}{c}\text { Autoria, ano de } \\
\text { publicação e local } \\
\text { do estudo }\end{array}$ & Título do artigo & Objetivo do estudo & $\begin{array}{l}\text { Delineamento } \\
\text { metodológico }\end{array}$ & Desfecho \\
\hline $\begin{array}{l}\text { Lima, A. S. et al. } \\
\text { 2018, Juiz de Fora. }\end{array}$ & $\begin{array}{c}\text { Análise da } \\
\text { prevalência da } \\
\text { Síndrome de } \\
\text { Burnout em } \\
\text { profissionais da } \\
\text { Atenção Primária } \\
\text { à Saúde }\end{array}$ & $\begin{array}{l}\text { Identificar a prevalência } \\
\text { de burnout nos } \\
\text { profissionais da atenção } \\
\text { primária e fatores } \\
\text { associados. }\end{array}$ & $\begin{array}{c}\text { Estudo } \\
\text { transversal. }\end{array}$ & $\begin{array}{l}\text { Influência que a SB tem sobre a } \\
\text { autoavaliação da condição de } \\
\text { saúde dos profissionais. Sabendo- } \\
\text { se que a SB causa exaustão física } \\
\text { e emocional nos indivíduos e } \\
\text { demais prejuízos à sua saúde. }\end{array}$ \\
\hline $\begin{array}{l}\text { Vidotti, V. et al. } \\
\text { 2018, Londrina. }\end{array}$ & $\begin{array}{c}\text { Síndrome de } \\
\text { Burnout e o } \\
\text { trabalho em } \\
\text { turnos na equipe } \\
\text { de enfermagem. }\end{array}$ & $\begin{array}{l}\text { Analisar os fatores } \\
\text { associados à síndrome } \\
\text { de Burnout, segundo o } \\
\text { turno de trabalho da } \\
\text { equipe de enfermagem. }\end{array}$ & $\begin{array}{c}\text { Estudo } \\
\text { transversal. }\end{array}$ & $\begin{array}{l}\text { Os fatores psicossociais e do } \\
\text { contexto laboral, sobretudo o } \\
\text { baixo apoio social, tiveram } \\
\text { associação com as dimensões da } \\
\text { síndrome entre os profissionais de } \\
\text { enfermagem de ambos os turnos. }\end{array}$ \\
\hline $\begin{array}{l}\text { Puerto, J. C. et al. } \\
\text { 2017, Espanha. }\end{array}$ & $\begin{array}{l}\text { Uma nova } \\
\text { contribuição para } \\
\text { a classificação dos } \\
\text { fatores estressores } \\
\text { que afetam os } \\
\text { profissionais de } \\
\text { enfermagem }\end{array}$ & $\begin{array}{l}\text { Identificar e classificar } \\
\text { os principais fatores } \\
\text { ocupacionais mais } \\
\text { estressantes que afetam } \\
\text { os profissionais de } \\
\text { enfermagem nas } \\
\text { unidades médicas do } \\
\text { hospital. }\end{array}$ & $\begin{array}{c}\text { Estudo quanti- } \\
\text { qualitativo, } \\
\text { descritivo e } \\
\text { prospectivo. }\end{array}$ & $\begin{array}{l}\text { Os fatores estressores de maior } \\
\text { consenso foram classificados, } \\
\text { sendo que os primeiros foram a } \\
\text { sobrecarga de trabalho, as } \\
\text { interrupções frequentes durante a } \\
\text { execução de suas tarefas, } \\
\text { trabalhar em horário noturno e, } \\
\text { finalmente, a simultaneidade em } \\
\text { executar tarefas diferentes. }\end{array}$ \\
\hline $\begin{array}{l}\text { Dutra, H. S. et al, } \\
\text { 2019, Juiz de Fora. }\end{array}$ & $\begin{array}{l}\text { Burnout entre } \\
\text { profissionais de } \\
\text { enfermagem em } \\
\text { hospitais do } \\
\text { Brasil. }\end{array}$ & $\begin{array}{l}\text { Avaliar a ocorrência } \\
\text { de Burnout entre os } \\
\text { profissionais de } \\
\text { enfermagem de três } \\
\text { hospitais públicos. }\end{array}$ & $\begin{array}{c}\text { Estudo } \\
\text { transversal. }\end{array}$ & $\begin{array}{l}\text { A exaustão emocional tem sido } \\
\text { caracterizada como central no } \\
\text { desenvolvimento } \\
\text { de Burnout entre profissionais de } \\
\text { enfermagem e a } \\
\text { despersonalização frequentemente } \\
\text { acompanha o sentimento de } \\
\text { exaustão emocional. }\end{array}$ \\
\hline $\begin{array}{l}\text { Bezerra, C. M. B. et } \\
\text { al. 2019, Natal. }\end{array}$ & $\begin{array}{l}\text { Prevalência do } \\
\text { estresse e } \\
\text { síndrome de } \\
\text { Burnout em } \\
\text { enfermeiros no } \\
\text { trabalho } \\
\text { hospitalar em } \\
\text { turnos. } \\
\end{array}$ & $\begin{array}{c}\text { Verificar o nível de } \\
\text { estresse e a existência } \\
\text { da síndrome } \\
\text { de Burnout em } \\
\text { enfermeiros nos turnos } \\
\text { diurno e noturno na área } \\
\text { hospitalar. }\end{array}$ & $\begin{array}{l}\text { Estudo descritivo } \\
\text { e analítico. }\end{array}$ & $\begin{array}{l}\text { O nível de estresse entre os } \\
\text { enfermeiros e as três dimensões } \\
\text { da síndrome foram avaliados } \\
\text { como nível médio nos turnos } \\
\text { diurno e noturno. Houve } \\
\text { correlação estatisticamente } \\
\text { significativa entre estresse } \\
\text { e Burnout. }\end{array}$ \\
\hline $\begin{array}{l}\text { Santos, E. N. et al. } \\
\text { 2019, São Paulo. }\end{array}$ & $\begin{array}{c}\text { Saúde do } \\
\text { trabalhador no } \\
\text { ambiente } \\
\text { hospitalar: fatores } \\
\text { de risco para a } \\
\text { síndrome de } \\
\text { Burnout } \\
\end{array}$ & $\begin{array}{l}\text { Identificar os fatores de } \\
\text { risco aos enfermeiros e } \\
\text { técnicos de enfermagem } \\
\text { no ambiente de trabalho } \\
\text { que possibilite a } \\
\text { síndrome de Burnout. }\end{array}$ & $\begin{array}{c}\text { Estudo analítico, } \\
\text { observacional e } \\
\text { quantitativo. }\end{array}$ & $\begin{array}{l}\text { A população estudada tem } \\
\text { apresentou esgotamento crítico. }\end{array}$ \\
\hline $\begin{array}{l}\text { Rodriguez, E. O. L. } \\
\text { et al. 2018, Aracaju. }\end{array}$ & $\begin{array}{c}\text { Estresse } \\
\text { ocupacional em } \\
\text { profissionais de } \\
\text { enfermagem }\end{array}$ & $\begin{array}{c}\text { Analisar os fatores de } \\
\text { estresse em enfermeiros } \\
\text { de um hospital } \\
\text { universitário da cidade } \\
\text { de Aracaju, Sergipe, } \\
\text { Brasil. }\end{array}$ & $\begin{array}{c}\text { Estudo descritivo } \\
\text { e correlacional. }\end{array}$ & $\begin{array}{c}\text { A enfermagem se revelou como } \\
\text { uma profissão de risco para o } \\
\text { estresse ocupacional, sofrendo } \\
\text { diversas influências dos fatores } \\
\text { analisados, relacionados à } \\
\text { organização e ao processo de } \\
\text { trabalho. }\end{array}$ \\
\hline $\begin{array}{l}\text { Soares, R. S. 2018, } \\
\text { Niterói. }\end{array}$ & $\begin{array}{l}\text { Burnout e fatores } \\
\text { associados entre } \\
\text { profissionais de } \\
\text { enfermagem do } \\
\text { hospital municipal } \\
\text { no Rio de Janeiro. }\end{array}$ & $\begin{array}{l}\text { Descrever a prevalência } \\
\text { e possíveis fatores } \\
\text { associados à síndrome } \\
\text { de burnout entre } \\
\text { profissionais de } \\
\text { enfermagem de setores } \\
\text { fechados. }\end{array}$ & $\begin{array}{c}\text { Estudo } \\
\text { observacional e } \\
\text { descritivo } \\
\text { seccional. }\end{array}$ & $\begin{array}{c}\text { Os prejuízos do burnout } \\
\text { extrapolam a esfera profissional, } \\
\text { podendo causar prejuízos na } \\
\text { qualidade de vida, com os } \\
\text { sintomas de depressão; distúrbios } \\
\text { gastrointestinais; } \\
\text { cardiovasculares; cognitivos; } \\
\text { flutuações de humor. }\end{array}$ \\
\hline $\begin{array}{l}\text { Mourão, A. L. et al. } \\
\text { 2017, Bahia. }\end{array}$ & $\begin{array}{l}\text { Síndrome de } \\
\text { Burnout no } \\
\text { contexto da }\end{array}$ & $\begin{array}{l}\text { Abordar as experiências } \\
\text { inerentes ao contexto } \\
\text { dos profissionais da }\end{array}$ & $\begin{array}{l}\text { Revisão crítico- } \\
\text { reflexiva. }\end{array}$ & $\begin{array}{l}\text { Transtornos por situações de } \\
\text { estresse no âmbito da } \\
\text { enfermagem, essa síndrome está }\end{array}$ \\
\hline
\end{tabular}




\begin{tabular}{|c|c|c|c|c|}
\hline & Enfermagem. & $\begin{array}{l}\text { enfermagem e suas } \\
\text { possíveis relações com } \\
\text { a síndrome de burnout. }\end{array}$ & & $\begin{array}{c}\text { presente como um transtorno } \\
\text { ocupacional que vem impactando } \\
\text { na prestação da assistência desses } \\
\text { trabalhadores. }\end{array}$ \\
\hline $\begin{array}{l}\text { Zavalis, A. et al. } \\
\text { 2015, Rio de } \\
\text { Janeiro. }\end{array}$ & $\begin{array}{l}\text { A influência dos } \\
\text { fatores estressores } \\
\text { sobre os níveis de } \\
\text { atenção de } \\
\text { profissionais de } \\
\text { enfermagem. }\end{array}$ & $\begin{array}{l}\text { Identificar, segundo a } \\
\text { referência dos } \\
\text { profissionais de } \\
\text { enfermagem, quais } \\
\text { fatores estressores têm a } \\
\text { maior influência no } \\
\text { processo de atenção; e } \\
\text { analisar quais geram } \\
\text { maior impacto no } \\
\text { desenvolvimento das } \\
\text { atividades de cuidado. }\end{array}$ & $\begin{array}{c}\text { Estudo } \\
\text { quantitativo. }\end{array}$ & $\begin{array}{l}\text { Fatores estressores relacionados } \\
\text { ao ambiente tiveram a maior } \\
\text { influência na atenção e } \\
\text { possivelmente são os que mais } \\
\text { interferem na qualidade da } \\
\text { assistência prestada aos clientes }\end{array}$ \\
\hline $\begin{array}{l}\text { Ribeiro, E. K. A. et } \\
\text { al. 2021, Campina } \\
\text { Grande. }\end{array}$ & $\begin{array}{c}\text { Influência da } \\
\text { síndrome de } \\
\text { Burnout na } \\
\text { qualidade de vida } \\
\text { de profissionais } \\
\text { de enfermagem: } \\
\text { estudo } \\
\text { quantitativo. } \\
\end{array}$ & $\begin{array}{l}\text { Estimar a prevalência e } \\
\text { os fatores associados à } \\
\text { síndrome de burnout e } \\
\text { qualidade de vida em } \\
\text { profissionais de } \\
\text { enfermagem. }\end{array}$ & $\begin{array}{l}\text { Estudo transversal } \\
\text { e analítico. }\end{array}$ & $\begin{array}{l}\text { A síndrome de burnout tem } \\
\text { influência no desfecho da } \\
\text { qualidade de vida dos } \\
\text { profissionais de enfermagem, } \\
\text { sendo mais prevalente entre } \\
\text { profissionais com maior idade, } \\
\text { alta renda e entre enfermeiros. }\end{array}$ \\
\hline $\begin{array}{l}\text { Fernandes, L. S. et } \\
\text { al. 2017, São Paulo. }\end{array}$ & $\begin{array}{l}\text { Síndrome de } \\
\text { burnout em } \\
\text { profissionais de } \\
\text { enfermagem de } \\
\text { uma unidade de } \\
\text { terapia intensiva }\end{array}$ & $\begin{array}{c}\text { Avaliar o nível da } \\
\text { Síndrome de Burnout } \\
\text { nos profissionais de } \\
\text { enfermagem na } \\
\text { Unidade de Terapia } \\
\text { Intensiva. }\end{array}$ & $\begin{array}{c}\text { Estudo } \\
\text { quantitativo e } \\
\text { transversal. }\end{array}$ & $\begin{array}{l}\text { O ambiente intensivista é propício } \\
\text { para o desenvolvimento da } \\
\text { Síndrome, bem como aqueles que } \\
\text { trabalhavam } 12 \text { horas diárias e os } \\
\text { não praticantes de atividade } \\
\text { física. }\end{array}$ \\
\hline $\begin{array}{l}\text { Silva, G. S. A. et al. } \\
\text { 2018, São Paulo. }\end{array}$ & $\begin{array}{l}\text { Estresse e burnout } \\
\text { em profissionais } \\
\text { de enfermagem de } \\
\text { uma unidade de } \\
\text { terapia intensiva e } \\
\text { semi-intensiva. }\end{array}$ & $\begin{array}{c}\text { Identificar a frequência } \\
\text { dos sintomas de estresse } \\
\text { e a ocorrência de } \\
\text { Síndrome de Burnout } \\
\text { em profissionais de } \\
\text { enfermagem que atuam } \\
\text { em unidade de terapia } \\
\text { intensiva e semi- } \\
\text { intensiva. }\end{array}$ & $\begin{array}{l}\text { Estudo analítico, } \\
\text { transversal e } \\
\text { quantitativo. }\end{array}$ & $\begin{array}{l}\text { Embora o ambiente de Terapia } \\
\text { Intensiva e Semi-Intensiva } \\
\text { contribua para o estresse, } \\
\text { predominam profissionais com } \\
\text { moderado estresse. }\end{array}$ \\
\hline $\begin{array}{c}\text { Silva, F. G. et al. } \\
\text { 2019, Ceará. }\end{array}$ & $\begin{array}{l}\text { Predisposição } \\
\text { para a síndrome } \\
\text { de burnout na } \\
\text { equipe de } \\
\text { enfermagem do } \\
\text { serviço de } \\
\text { atendimento } \\
\text { móvel de } \\
\text { urgência. }\end{array}$ & $\begin{array}{c}\text { Conhecer a } \\
\text { predisposição para a } \\
\text { Síndrome de Burnout na } \\
\text { equipe de Enfermagem } \\
\text { do Serviço de } \\
\text { Atendimento Móvel de } \\
\text { Urgência (SAMU). }\end{array}$ & $\begin{array}{l}\text { Pesquisa } \\
\text { exploratória e } \\
\text { descritiva. }\end{array}$ & $\begin{array}{l}\text { A equipe de enfermagem do } \\
\text { SAMU tem predisposição a } \\
\text { Síndrome de Burnout. }\end{array}$ \\
\hline
\end{tabular}

Fonte: Autores (2021).

As publicações se concentram no ano de 2018, que dispôs de 5 artigos (35,7\%), seguido do ano de 2019, que possui 4 publicações (28,5\%). O ano de 2017 apresentou 3 artigos (21,4\%), seguido do ano de 2015 e 2021 ambos apresentam 1 artigo (7,1\%). Assim, os anos de 2016 e 2020 não apresentaram publicações.

A análise do corpus resultou na construção de 4 categorias de discussão, que estão dispostas no Quadro 3 a partir dos artigos relacionados e suas respectivas base de dados. 
Quadro 3: Categorização dos dados a partir da análise de conteúdo. Brasil, 2021.

\begin{tabular}{|c|c|c|}
\hline Categoria & Título do artigo & Base de dados \\
\hline \multirow{8}{*}{ Sobrecarga de trabalho } & $\begin{array}{l}\text { Estresse e burnout em profissionais } \\
\text { de enfermagem de uma unidade de } \\
\text { terapia intensiva e semi-intensiva. }\end{array}$ & LILACS \\
\hline & $\begin{array}{c}\text { Predisposição para a síndrome de } \\
\text { burnout na equipe de enfermagem } \\
\text { do serviço de atendimento móvel de } \\
\text { urgência. }\end{array}$ & LILACS \\
\hline & $\begin{array}{c}\text { Análise da prevalência da Síndrome } \\
\text { de Burnout em profissionais da } \\
\text { Atenção Primária à Saúde }\end{array}$ & SciELO \\
\hline & $\begin{array}{c}\text { Burnout entre profissionais de } \\
\text { enfermagem em hospitais do Brasil. }\end{array}$ & SciELO \\
\hline & $\begin{array}{l}\text { Uma nova contribuição para a } \\
\text { classificação dos fatores estressores } \\
\text { que afetam os profissionais de } \\
\text { enfermagem }\end{array}$ & SciELO \\
\hline & $\begin{array}{l}\text { Prevalência do estresse e síndrome } \\
\text { de Burnout em enfermeiros no } \\
\text { trabalho hospitalar em turnos. }\end{array}$ & SciELO \\
\hline & $\begin{array}{l}\text { Burnout e fatores associados entre } \\
\text { profissionais de enfermagem do } \\
\text { hospital municipal no Rio de } \\
\text { Janeiro. }\end{array}$ & SciELO \\
\hline & $\begin{array}{c}\text { Síndrome de burnout em } \\
\text { profissionais de enfermagem de } \\
\text { uma unidade de terapia intensiva }\end{array}$ & LILACS \\
\hline \multirow{11}{*}{ Ambiente de trabalho } & $\begin{array}{l}\text { Estresse e burnout em profissionais } \\
\text { de enfermagem de uma unidade de } \\
\text { terapia intensiva e semi-intensiva. }\end{array}$ & LILACS \\
\hline & $\begin{array}{c}\text { Predisposição para a síndrome de } \\
\text { burnout na equipe de enfermagem } \\
\text { do serviço de atendimento móvel de } \\
\text { urgência. }\end{array}$ & LILACS \\
\hline & $\begin{array}{c}\text { Síndrome de Burnout e o trabalho } \\
\text { em turnos na equipe de } \\
\text { enfermagem. }\end{array}$ & SciELO \\
\hline & $\begin{array}{l}\text { Saúde do trabalhador no ambiente } \\
\text { hospitalar: fatores de risco para a } \\
\text { síndrome de Burnout }\end{array}$ & LILACS \\
\hline & $\begin{array}{l}\text { A influência dos fatores estressores } \\
\text { sobre os níveis de atenção de } \\
\text { profissionais de enfermagem. }\end{array}$ & LILACS \\
\hline & $\begin{array}{l}\text { Burnout e fatores associados entre } \\
\text { profissionais de enfermagem do } \\
\text { hospital municipal no Rio de } \\
\text { Janeiro. }\end{array}$ & SciELO \\
\hline & $\begin{array}{c}\text { Análise da prevalência da Síndrome } \\
\text { de Burnout em profissionais da } \\
\text { Atenção Primária à Saúde }\end{array}$ & SciELO \\
\hline & $\begin{array}{l}\text { Estresse e burnout em profissionais } \\
\text { de enfermagem de uma unidade de } \\
\text { terapia intensiva e semi-intensiva. }\end{array}$ & LILACS \\
\hline & $\begin{array}{c}\text { Burnout entre profissionais de } \\
\text { enfermagem em hospitais do Brasil. }\end{array}$ & SciELO \\
\hline & $\begin{array}{c}\text { Estresse ocupacional em } \\
\text { profissionais de enfermagem }\end{array}$ & SciELO \\
\hline & $\begin{array}{l}\text { Síndrome de Burnout no contexto } \\
\text { da Enfermagem. }\end{array}$ & LILACS \\
\hline
\end{tabular}




\begin{tabular}{|c|c|c|}
\hline \multirow{1}{*}{ Desgaste emocional } & $\begin{array}{c}\text { Burnout e fatores associados entre } \\
\text { profissionais de enfermagem do } \\
\text { hospital municipal no Rio de } \\
\text { Janeiro. }\end{array}$ & SciELO \\
\cline { 2 - 3 } & $\begin{array}{c}\text { Influência da síndrome de Burnout } \\
\text { na qualidade de vida de } \\
\text { profissionais de enfermagem: } \\
\text { estudo quantitativo. }\end{array}$ & SciELO \\
\cline { 2 - 3 } & $\begin{array}{c}\text { Predisposição para a síndrome de } \\
\text { burnout na equipe de enfermagem } \\
\text { do serviço de atendimento móvel de } \\
\text { urgência. }\end{array}$ & LILACS \\
\hline \multirow{5}{*}{$\begin{array}{c}\text { Desvalorização } \\
\text { profissional }\end{array}$} & $\begin{array}{c}\text { Estresse e burnout em profissionais } \\
\text { de enfermagem de uma unidade de } \\
\text { terapia intensiva e semi-intensiva. }\end{array}$ & LILACS \\
\cline { 2 - 3 } & $\begin{array}{c}\text { Burnout entre profissionais de } \\
\text { enfermagem em hospitais do Brasil. }\end{array}$ & SciELO \\
\cline { 2 - 3 } & $\begin{array}{c}\text { Estresse ocupacional em } \\
\text { profissionais de enfermagem }\end{array}$ & SciELO \\
\cline { 2 - 3 } & $\begin{array}{c}\text { Síndrome de Burnout e o trabalho } \\
\text { em turnos na equipe de } \\
\text { enfermagem. }\end{array}$ & SciELO \\
\hline
\end{tabular}

Fonte: Autores (2021).

A categoria que concentrou a maioria das publicações foi Sobrecarga de Trabalho composta por 8 artigos. A categoria de Ambiente de trabalho e Desgaste emocional ambas foram compostas por 7 publicações cada, seguida de Desvalorização profissional com 4 artigos.

\section{Discussão}

Os profissionais de enfermagem apresentaram médio nível de estresse 48\%, apresentando sentimentos de sobrecarga de trabalho, cansaço e desgaste sendo assim os sintomas mais frequentes para desencadear a SB (Silva et al., 2018).

Em outro estudo, $26 \%$ da classe de profissionais da enfermagem apresentaram exaustão no trabalho. Tal desgaste é sinal de uma disfunção importante no ambiente de trabalho, portanto revela mais sobre o local de trabalho do que sobre o que eles trabalham (Silva et al., 2018).

Os profissionais de enfermagem que estão sob regime de trabalho estatutário sendo regidos por leis trabalhistas que diferem entre si de acordo com férias, remuneração e benefícios. Assim, essas discrepâncias pode ser um fator desencadeador da SB devido a produção de um ambiente organizacional com ênfase na cooperação, participação e integração das atividades assistenciais (da Silva et al., 2019).

Além disso, o regime de trabalho de 12 horas influencia no desenvolvimento da SB, visto que a carga de trabalho acarreta sentimentos de não realização eficiente do trabalho e distanciamento do paciente. Isso se dá, devido ao número reduzido de profissionais escalados com relação a demanda de trabalho solicitada (da Silva et al., 2019).

Foi observado que 39,7\% dos profissionais possuíam dois ou mais vínculos de trabalho sendo uma busca de incremento na renda familiar, destes $34,5 \%$ eram técnicos e auxiliares de enfermagem. É preciso ressaltar que trabalhar em regimes de plantão além das quarenta horas regulares afeta o organismo pelas noites de sono perdidas e pela maior sobrecarga de trabalho (Lima \& Farah, 2018).

Os trabalhadores de enfermagem devem se submeter ao trabalho em turnos para garantir a continuidade do atendimento 24 horas por dia e todos os dias da semana com cuidados prestados aos pacientes. Contudo, o trabalho em turnos tem sido associado a diversas alterações nas funções biológicas que conduzem ao agravo físico e mental. Assim, foi 
evidenciado que os fatores associados à exaustão emocional estão ligados com a carga de trabalho relacionando negativamente a síndrome (Puerto et al., 2017).

As avaliações do acometimento da SB nos profissionais de enfermagem são essenciais visto que no momento em que o trabalhador é acometido por uma doença ele possui seu desempenho afetado em consequência da mesma, e com isso seus colegas de trabalho irão conviver com uma sobrecarga de trabalho elevada devido ao baixo rendimento do colaborador afetado. Com isso, se tem carga de trabalho elevada aumentando o risco de possuir burnout causando consequências mais amplas a equipe (Dutra et al., 2019).

As características intrínsecas do trabalho exercido e do trabalhador com sobrecargas psíquicas também são fatores agravantes da SB. Pois acredita-se que o trabalho estressante, cobranças, obrigações e conflitos permanentes particularmente na enfermagem por lidar com o cuidado específicos em situações de sofrimento, exigências de mais competências interpessoais acarretando assim o burnout (Bezerra, 2016).

A sobrecarga pode ocasionar relações interpessoais conflituosas e com isso se tem profissionais expostos a fatores considerados como estressores e que conduzem ao esgotamento físico e emocional. Assim, a enfermagem mostra-se como uma das profissões com enormes possibilidades de desenvolver a SB considerando a sobrecarga de trabalho, falta de autonomia e o grande contato com pacientes e familiares que interferem de forma negativa (Bezerra, 2016).

Em alguns estudos foi relatado que a SB está associada aos problemas no ambiente de trabalho do que individuais, visto que o mesmo influencia na forma como os indivíduos enxergam o trabalho se ele é prazeroso ou não, e como lidam com ele e suas próprias vidas associando-se assim ao burnout (Lima \& Farah, 2018).

A Unidade de Terapia Intensiva (UTI) de acordo com os profissionais de enfermagem atuantes no setor $57,1 \%$ consideram como um lugar estressante devido a intensa proximidade com pacientes de alta complexidade e $23,8 \%$ apresentaram altos níveis de estresse. Tais achados devem ser considerados devido o estresse aumentando assim o risco de negligência e comprometimento da qualidade assistencial sendo também um fator que aumenta o risco do desenvolvimento do mesmo podendo desencadear a SB (Silva et al., 2018).

De acordo com a Previdência Social no Brasil em uma pesquisa feita no período de 2012 a 2016, foram afastados 34.511 diagnosticados com SB. Dessa forma, ela se mostrou mais prevalente nos técnicos de enfermagem podendo ser explicado por elementos específicos como o constante medo de cometer erros no cuidado ao paciente, ausência de incentivos no desenvolvimento profissional e falta de valorização profissional. Assim, são fatores que podem predispor nos técnicos de enfermagem um maior risco de possuir a Síndrome (Vidott et al., 2018).

Os profissionais de enfermagem atuantes no Serviço de Atendimento Móvel de Urgência (SAMU) estão submetidos continuamente a riscos tanto de nível físico como psíquico por se tratar de um ambiente permeado de imprevisibilidade além de lidar com risco iminente de morte. Com isso, na zona urbana enfrentam diariamente o trânsito caótico, má sinalização e buracos nas ruas além do seu trabalho em áreas de risco e do complicado relacionamento com a população, equipe de trabalho e chefia (da Silva et al., 2019).

A equipe de enfermagem que atua diretamente com a Atenção Primária a Saúde (APS) estão mais propensos a desenvoltura da SB visto que, seu ambiente de trabalho possui contato direto com as pessoas e seus sofrimentos sentindo-se cobrados e pressionados a dar respostas as necessidades da comunidade que atendem. Além de ser cobrados de produtividade em um ambiente que possui carência de recursos humanos, infraestrutura e materiais (Lima \& Farah, 2018).

O fator estressor que possuiu maior relevância para o desencadeamento da SB tem sido a sobrecarga de trabalho estando relacionadas as Unidades de Terapia Intensiva, Emergência, Atenção Primária e Serviços Especializados. Além disso, esse estresse pode ser desencadeado devido ao ambiente físico de trabalho, exigências do próprio trabalho e conteúdo do trabalho (Lima \& Farah, 2018). 
Os participantes desse estudo sendo eles os profissionais de enfermagem apresentaram sintomas como dor no peito, pressão arterial alto, excesso ou perda de apetite além da dificuldade com o sono sendo todos os fatores relacionados ao desgaste no geral devido ao ambiente de trabalho. Assim, sendo constatado que 17,6\% dos enfermeiros associaram problemas de saúde devido o trabalho no hospital (Santos et al., 2019).

Os profissionais que trabalham em ambientes considerados críticos, como a UTI apresentam uma alta predisposição para ser acometido pelo sofrimento psíquico tendo em vista a complexidade das ações ali realizadas, causando estresse gerado durante a sua realização e a ocorrência de morte de pacientes. O ambiente da UTI pode ser considerado um ambiente exaustivo, sobrecarregado e agressivo caracterizado como um ambiente tenso, local onde a morte se torna constante (Soares, 2018).

A estrutura organizacional da instituição hospitalar possui sua parcela na ocorrência de estresse, interferindo na vida pessoal e profissional do indivíduo. Por muitas vezes, trabalhar em condições insalubres e inseguras influencia diretamente no bem-estar físico e psíquico do indivíduo. Além disso, a falta suficiente de profissionais é fonte considerável de estresse, repercutindo assim na qualidade do atendimento (Zavalis et al., 2015).

O desenvolvimento da SB é um processo gradual de desgaste, acompanhando de sintomas físicos e psíquicos. Ele pode ser compreendido como a falta de energia juntamente a um sentimento de esgotamento emocional, observa-se que de acordo com os profissionais de enfermagem $44 \%$ apresentam alto desgaste emocional. Sinalizando assim, uma possibilidade futura de desenvolvimento da SB que demanda medidas preventivas (Zavalis et al., 2015).

Em outro estudo foi encontrado que 30,5\% dos profissionais de enfermagem apresentaram nível elevado de exaustão emocional. Com isso, especificamente as demandas emocionais relacionadas ao trabalho podem interferir no desempenho do mesmo diante das necessidades dos pacientes. Podendo ser observado o distanciamento social e cognitivo em relação ao trabalho como uma forma de lidar com a situação (Dutra et al., 2019).

Quanto ao curto prazo para cumprir ordens relacionadas ao trabalho, estudos sobre o estresse e distúrbios psíquicos nos trabalhadores de enfermagem foi encontrado que a velocidade para a realização das ações e o tempo geram pressões psicológicas e, portanto, predispondo o desencadeamento da SB (Santana et al., 2020).

Foi encontrado que a organização no trabalho exercia uma forte influência sobre o aparo psíquico dos profissionais de enfermagem podendo ocorrer após experiências negativas o desgaste gradual do sujeito resultando em um quadro sintomatológicos como a presença de ansiedade, depressão, cefaleia, agitação e irritabilidade. Assim é válido destacar que os sintomas aparecem de forma distinta em cada trabalhador (Mourao et al., 2017).

O estado emocional dos profissionais que trabalham em setores fechados altera-se no decorrer do plantão visto que pode estar associado ao desgaste e estresse da atividade de fornecer ao paciente assistência e cuidado, principalmente devido a existência de altos níveis de habilidade e conhecimento que exige resposta imediata (Soares, 2018).

Nesse estudo, foi encontrado que técnicos e auxiliares de enfermagem revelaram maiores chances de baixa valorização profissional devido sua menor autonomia em comparação aos demais membros da equipe de saúde sendo assim pouco reconhecidos e valorizados o que pode gerar um sentimento de inutilidade e incompetência apesar da relevância do seu trabalho (Soares, 2018).

Deve se destacar a dificuldade de reconhecimento, valorização e obtenção de resultados quando se trata de processos de trabalho em equipe como é o caso da Enfermagem, sendo assim difícil para cada profissional bem como para os pacientes e suas famílias identificar a contribuição individual de cada profissional no resultado final da prestação da assistência (Dutra $e t$ al., 2019).

Em sintonia com os resultados anteriores, estudo sobre tensões ocupacionais na enfermagem revelou que o baixo salário foi considerado como fonte de estresse devido principalmente ao cumprimento da jornada dupla de trabalho. Com isso, 
o ritmo de trabalho acelerado, as dobras de plantão, existência de salários injustos incompatíveis com a realidade e a sobrecarga trabalhista pode afetar o desempenho físico, emocional e mental (Santana et al., 2020).

\section{Considerações Finais}

A pesquisa documental permitiu observar que, existem fatores que indubitavelmente, potencializam a incidência da Síndrome de Burnout nos profissionais de enfermagem prejudicando assim, sua assistência e comprometendo sua saúde física, mental e psíquica. Os achados deste artigo mostram a necessidade de acompanhamento das condições de trabalho e saúde destes profissionais, além disso é preciso que se tenha uma implementação de estratégias que possam minimizar os danos à saúde dos mesmos.

\section{Referências}

Bezerra, C. M. B. (2016). Estresse e síndrome de Burnout nos enfermeiros de um hospital universitário (Master's thesis, Brasil).

da Silva, F. G., de Paulo Andrade, A., de Azevedo Ponte, K. M., Ferreira, V. E. S., da Silva Sousa, B., \& Gonçalves, K. G. (2019). Predisposição para síndrome de burnout na equipe de enfermagem do serviço de atendimento móvel de urgência. Enfermagem em Foco, 10(1).

Dutra, H. S., Gomes, P. A. L., Garcia, R. N., Oliveira, H. C., de Freitas, S. C., \& de Brito Guirardello, E. (2019). Burnout entre profissionais de enfermagem em hospitais no Brasil. Revista Cuidarte, 10(1).

Fernandes, L. S., Nitsche, M. J. T., \& de Godoy, I. (2017). Síndrome de burnout em profissionais de enfermagem de uma unidade de terapia intensiva Burnout syndrome in nursing professionals from an intensive care unit. Revista de Pesquisa Cuidado é Fundamental Online, 9(2), 551-557.

Lima, A. D. S., Farah, B. F., \& Bustamante-Teixeira, M. T. (2017). Análise da prevalência da síndrome de burnout em profissionais da atenção primária em saúde. Trabalho, Educação e Saúde, 16, 283-304.

Ludke, M. \& Andre, M. E. D. A. (2013). Pesquisas em educação: uma abordagem qualitativa. E.P.U.

Mourão, A. L., de Carvalho Costa, A. C., Silva, E. M. M., \& Lima, K. J. (2017). Síndrome de Burnout no contexto da enfermagem. Revista baiana de saúde pública, 41(1).

Perniciotti, P., Serrano Júnior, C. V., Guarita, R. V., Morales, R. J., \& Romano, B. W. (2020). Síndrome de Burnout nos profi ssionais de saúde: atualização sobre definições, fatores de risco e estratégias de prevenção. Revista da SBPH, 23(1), 35-52.

Puerto, J. C., Soler, L. M., Montesinos, M. J. L., Marcos, A. P., \& Chorda, V. M. G. (2017). A new contribution to the classification of stressors affecting nursing professionals. Revista latino-americana de enfermagem, 25.

Santana, L. C., Ferreira, L. A., \& Santana, L. P. M. (2020). Occupational stress in nursing professionals of a university hospital. Revista Brasileira de Enfermagem, 73 .

Santos, E. N. D., Boas, L. L. V., Miranda, A. P., dos Santos, E. N., de França, Í. J. S., Boas, L. L. V., \& Miranda, A. P. (2019). Saúde do trabalhador no ambiente hospitalar: fatores de risco para síndrome de burnout. Nursing (Säo Paulo), 2572-2576.

Silva, B. M. D., Lima, F. R. F., Farias, F. S. D. A. B., \& Campos, A. D. C. S. (2006). Jornada de trabalho: fator que interfere na qualidade da assistência de enfermagem. Texto \& Contexto-Enfermagem, 15, 442-448.

Silva, G. D. S. A. D., Silva, G. A. V. D., Silva, R. M. D., Andolhe, R., Padilha, K. G., \& Costa, A. L. S. (2018). Estresse e burnout em profissionais de enfermagem de unidade de terapia intensiva e semi-intensiva. Revista de Divulgação Científica Sena Aires, 7(1), 5-11.

Soares, R. D. S. (2018). Burnout e fatores associados entre profissionais de enfermagem de hospital municipal no Rio de Janeiro.

Sousa, M. B. C. D., Silva, H. P. A., \& Galvão-Coelho, N. L. (2015). Stress response: I. Homestasis and allostasis theory. Estudos de Psicologia (Natal), 20(1), 2-11.

Souza, M. T. D., Silva, M. D. D., \& Carvalho, R. D. (2010). Revisão integrativa: o que é e como fazer. Einstein (São Paulo), 8, 102-106.

Vidotti, V., Ribeiro, R. P., Galdino, M. J. Q., \& Martins, J. T. (2018). Síndrome de Burnout y trabajo en turnos en el equipo de enfermería1. Revista LatinoAmericana de Enfermagem, 26.

Vieira, I., \& Russo, J. A. (2019). Burnout e estresse: entre medicalização e psicologização. Physis: Revista de Saúde Coletiva, 29 , e290206.

Zavalis, A., Vianna, L. A. M., de Souza Velasque, L., Schutz, V., \& Machado, D. A. (2015). The influence of stress factors on the attention levels of nursing professionals. Revista de Pesquisa Cuidado é Fundamental Online, 7(4), 3375-3387.

Zoltowski, A. P. C., Costa, A. B., Teixeira, M. A. P., \& Koller, S. H. (2014). Qualidade metodológica das revisões sistemáticas em periódicos de psicologia brasileiros. Psicologia: teoria e Pesquisa, 30, 97-104. 